\title{
Simpler dental surgery should be done locally
}

Experts have called for an investigation into why more simple surgery, such as wisdom tooth removal, is not being done outside of hospital to help free up resources.

There should also be greater efforts to develop the 'hub and spoke' approach to oral and maxillofacial surgery, to ensure patients receive the best possible care according to their needs.

These suggestions were made in the latest national report ${ }^{1}$ from the Getting It Right First Time (GIRFT) programme - a national programme funded by the Department of Health and Social Care and jointly overseen by NHS Improvement and the Royal National Orthopaedic Hospital NHS Trust which is designed to improve medical care within the NHS by reducing unwarranted variations.

The report, a review into oral and maxillofacial surgery across England published on 28 November 2018, was led by Consultant Oral and Maxillofacial Surgeon Maire Morton, who reviewed the data for 76 hospital trusts.

The review team visited more than 200 sites, meeting more than 2,000 surgeons, clinicians, support staff and trust managers.

The report says that many oral and maxillofacial units are carrying out high volumes of dentoalveolar surgery (including wisdom tooth removal), adding: 'We believe the volumes are so high that resources are not being used optimally and that a significant proportion of dentoalveolar surgery could be carried out in a more appropriate setting.

'A number of complex issues are pushing patients towards secondary care. These include patients not accessing primary care dentistry frequently enough (possibly due to cost and anxiety), leading to patients being referred directly to secondary care by their GP.

'Depending on the level of treatment complexity, the type of anaesthetic required and whether there are any comorbidities, a higher proportion of dentoalveolar surgery could be done as a Level 2 (intermediate care) procedure.'

The review report also outlines the need to centralise services and build on existing 'hub and spoke' networks for the specialty, especially in the treatment of patients with head and neck cancer and those needing corrective jaw surgery.

This configuration would enable central hubs to focus on in-patient work, while outlying spoke hospitals took care of day cases and out-patients, which formed a high proportion of oral and maxillofacial work.

Another recommendation of the report is to urge providers to consider allocating dedicated oral and maxillofacial theatre time for emergency cases, which in turn would help prevent out-of-hours operations and the cancellation of planned procedures, and reduce pre and post-operative length of hospital stay.

It also calls for a standard protocol to be introduced across the country to reduce unwarranted variations in the number of hospital follow-up appointments after surgery.
The improvement of data quality is also highlighted in the report. Morton and the GIRFT team identified 127 hospitals carrying out oral and maxillofacial surgery in England but found data-gathering problematic. There was a lack of consistency in coding and no comprehensive set of clinical outcome measures for oral and maxillofacial surgery.

If implemented, all of these steps could improve the patient experience through shorter hospital stays, fewer admissions and fewer repeat visits, which could also result in cost efficiencies of up to $£ 25$ million, says the report.

Morton said: 'Our review has shone a light onto the specialty and allowed us to discuss the difficult issues that underlie variation in care pathways in oral and maxillofacial surgery, some of which add cost while adding no value to the individual patient.'

British Association of Oral and Maxillofacial Surgeons (BAOMS) President Ian Martin said: 'The GIRFT review of oral and maxillofacial surgery has demonstrated significant opportunities to improve patient care with better data quality and data collection. It is crucial that oral and maxillofacial surgeons have access to good quality and timely data to deliver high-quality, patient-focused services.'

1. Getting It Right First Time (GIRFT). Oral and Maxillofacial Surgery: GIRFT Programme National Specialty Report. 2018. Available at http://gettingitrightfirsttime.co.uk/omfs-report/ (accessed December 2018).

\section{PEC election winners announced}

The people in charge of steering the BDA's strategy, priorities and finances for the next three years have been announced.

The results of the winners of the recently held BDA Principal Executive Committee (PEC) elections were announced on 11 December 2018.

The successful candidates from the election for the 2019-21 triennium were:
- Northern: Paul Blaylock

- Northern Ireland: Peter Crooks

- South West: Nigel Jones

- Yorkshire and Humber: Mick Armstrong

- UK-wide: Paul Woodhouse.

All of the people will take up their mandate on 1 January 2019 alongside the other ten sitting members.
The PEC - the BDA's board of directors - determines the organisation's strategic direction, priorities and finances to ensure all of the union members' money is reinvested into providing advice and support for all members.

Elections to the BDA's board take place every autumn with a third of all seats (five) available for nominations. 